\title{
Situs Ambiguous with Intestinal Malrotation, Short Pancreas and Interruption of The Inferior Vena Cava Revealing A Polysplenia Syndrome
}

\author{
Sara Dehbi ${ }^{*}$, Mohammed Aitchtouk, Ibtissam Zouita, Dounia Basraoui, Hicham Jalal
}

Department of Radiology, Mother-child Hospital, Med VI University Center, Marrakech, Morocco

DOI: $10.36347 /$ simcr.2020.v08i02.034

| Received: 03.02.2020 | Accepted: 17.02.2020 | Published: 27.02.2020

*Corresponding author: Dehbi Sara

Abstract

Case Report

Polysplenia syndrome is a rare situs ambiguous anomaly associated with multiple spleens and anomalies of chest and abdominal organs. We present a case of polysplenia syndrome found in a 11-year-old child. Radiological examinations are helpful to the diagnosis by showing the location and aspect of spleen, location of other organs in the abdomen, and identification of other associated anomalies. In this article, we illustrate anomalies of polysplenia syndrome and focus on abdominal organs.

Keywords: Imaging, Polysplénia, Situs ambigus, Abdomen.

Copyright @ 2020: This is an open-access article distributed under the terms of the Creative Commons Attribution license which permits unrestricted use, distribution, and reproduction in any medium for non-commercial use (NonCommercial, or CC-BY-NC) provided the original author and source are credited.

\section{INTRODUCTION}

Polysplenia syndrome is a rare congenital anomaly of situs ambiguous associated with multiple spleens and midline location of abdominal organs or in rare cases a single bilobed spleen. During embryogenesis, the spleen plays an important role in the normal development and lateralization of visceral organs. Therefore, anomalies of the spleen are associated with many congenital anomalies of other organs and show wide spectrum of abnormalities in the abdominal organs $[1,2]$. Technical advances in sonography, computed tomography, and magnetic resonance imaging have greatly enhanced our ability to detect and characterize these anomalies [3].

\section{CaSe Report}

A 11-year-old child patient was admitted in our radiology department for tuberculous pleural effusion.

Computed tomography shows a right pulmonary condensation with fluid pleural effusion and the evidence of liver, gallbladder, and common bile duct on the middle of the abdomen. Stomach and a bilobed spleen were on the right side. It has also showed a common mesentery type 1A. An Inferior vena cava (IVC) interruption with azygous continuation has also been with a supra hepatic veins flow directly to the OG, short median pancreas and a preduodenal portal vein. The diagnosis of polysplenia syndrome was made. In addition to the discovery of this entity, the particularity of our case is that we had a single lobulated spleen that had been reported in a rare case of polysplenia syndrome.
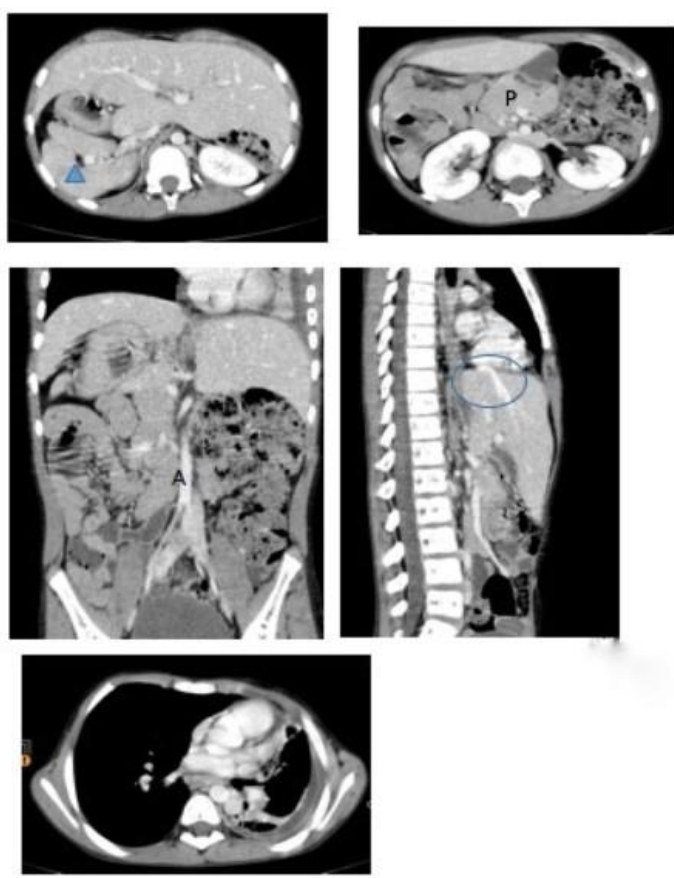

Fig. Axial CT images of the abdomen shows the liver on the middle with the lobulated spleen (triangle) stomach are on the right side, Inferior vena cava interruption with azygous continuation, a short pancreas (P) Coronal CT image : intestinal nonrotation. The descending aorta $(A)$ is on the right. Sagittal CT image showing supra hepatic veins flow directly to the OG (circle). 


\section{DISCUSSION}

Polysplenia syndrome refers to the association of 2 or more multiple spleens with multiple congenital abnormalities in abdomen and chest. However, some cases of polysplenia syndrome have been described with a single lobulated spleen or a single normal spleen. In all cases, the spleens are still located on the same side of the stomach [4].

It is a rare congenital disease initially described by Helwig in 1929 [3, 5]. Since then, few cases were described in the literature with an incidence of 1/250,000 live births [6].

Although situs ambiguous with polysplenia is associated with multiple spleens in the majority of patients, some studies report patients with situs ambiguous who have a single, lobulated spleen or even a normal spleen. In our case we had a single lobulated spleen located on the right side [7].

The pancreas may be normal, short in size, or have a cleft created by traversing vessels [2, 9, 10 ]. An annular pancreas and semiannular pancreas also have been reported 11 . The most often is a short pancreas with agenesis of the dorsal portion [4].

Polysplenia is commonly associated with anomalies of intestinal rotation. Intestinal rotation abnormalities include nonrotation (the small bowel is entirely on the right of the spine and the colon on the left), incomplete rotation (the appearance is between normal and non-rotation), and the rare reversed complete or incomplete rotation $[2,12]$.

Although gastrointestinal tract rotational anomalies may be a rare cause of abdominal pain in adults and may cause bowel obstruction, such as midgut volvulus [12].

A preduodenal portal vein was reported as one of the anomalies in association with polysplenia, is a rare anomaly resulting from maldevelopement of portal venous système or malposition of the abdominal vescera [14]

Inferior vena cava interruption with azygous continuation is the second most common abnormality observed in polysplenia patients after multiple spleens [4].

\section{CONCLUSION}

Polysplenia syndrome is a complex syndrome with a broad spectrum of abnormalities, the most common of which are multiple spleens and IVC interruption with azygous continuation. A CT-scan and reconstruction is excellent in visualizing these anomalies. Because the wide spectrum of anomalies in polysplenia syndrome do not have pathognomonic radiologic features, the knowledge of various radiologic features plays an important role in the diagnosis of this rare syndrome. In addition, correct diagnosis may decrease the mortality and morbidity.

\section{REFERENCES}

1. Muneta S, Sakai S, Fukuda H, Imamura Y, Matsumoto I. Polysplenia syndrome with various visceral anomalies in an adult: embryological and clinical considerations. Intern Med. 1992;31:10261031

2. Fulcher AS, Turner MA. Abdominal manifestations of situs anomalies in adults. Radiographics. 2002;22:1439-1456

3. Applegate KE, Goske MJ, Pierce G, Murphy D. Situs revisited: imaging of the heterotaxy syndrome. Radiographics. 1999;19(4):837-52.

4. Fulcher AS, Turner MA. Abdominal manifestations of situs anomalies in adults. Radiographics. 2002;22(6):1439-56.

5. Chen SJ, Li YW, Chang CI, Hsieh SC, Su CT, Hsu JCY. Usefulness of electron beam computed tomography in children with heterotaxy syndrome. Am J Cardiol. 1998;81(2):188-94

6. Rasool F, Mirza B. Polysplenia syndrome associated with situs inversus abdominus and type I jejunal atresia. APSP J Case Rep. 2011;2(2):18

7. Durmaz MS, Cengiz A, Arslan S, Erdogan H, Tolu I, Cengiz A. An incidental ndings of polysplenia syndrome in an adult patient with multiple anomalies. Clin Med Rev Case Rep. 2016;3:148

8. Maier M, Wiesner W, Mengiardi B. Annular pancreas and agenesis of the dorsal pancreas in a patient with polysplenia syndrome. Am J Roentgenol. 2007;188(2):W150-3

9. Gayer G, Apter S, Jonas T, Amitai M, Zissin R, Sella T. Polysplenia syndrome detected in adulthood: report of eight cases and review of the literature. Abdom Imaging. 1999;24:178-184

10. Kobayashi H, Kawamoto S, Tamaki T, Konishi J, Togashi K. Polysplenia associated with semiannular pancreas. Eur Ra- diol. 2001;11:16391641

11. Maier M, Wiesner W, Mengiardi B. Annular pancreas and agenesis of the dorsal pancreas in a patient with polysple- nia syndrome. AJR Am J Roentgenol. 2007;188:W150-W153

12. Peoples WM, Moller JH, Edwards JE. Polysplenia: a review of 146 cases. Pediatr Cardiol. 1983;4:129-137

13. Ditch eld MR, Hutson JM. Intestinal rotational abnormalities in polysplenia and asplenia syndromes. Pediatr Radiol. 1998;28:303-306

14. Pre-duodenal portal vein in polysplenia syndrome: clinical effects and surgical application. Surg Radiol Anat. 2011;33(5):451-4. 